\title{
Human Small Cell Lung Carcinoma and Carcinoid Tumor Regulate Dendritic Cell Maturation and Function
}

\author{
Nora S. Katsenelson, Galina V. Shurin, Svetlana N. Bykovskaia, Jeffrey Shogan, Michael R. Shurin \\ Department of Pathology (NSK, GVS, MRS), University of Pittsburgh Medical Center, University of \\ Pittsburgh Cancer Institute, Pittsburgh, Pennsylvania; and Bone Marrow Transplantation Program (SNB, \\ JS), Allegheny General Hospital, Pittsburgh, Pennsylvania
}

The induction of apoptosis in dendritic cells (DC) is a key mechanism by which tumors escape immune recognition and elimination. In fact, a number of studies have showed the correlation between the number of DC within the tumor and the clinical prognosis, suggesting that increased infiltration of tumor tissue by DC was associated with better patient survival and low incidence of metastatic disease. We compared the number of DC and their distribution pattern in human small-cell lung carcinoma and bronchial carcinoid tumor (CT) tissues. Immunohistochemical analysis revealed the presence of cells expressing DC markers CD1a and CD83 in small-cell lung carcinoma tissues and the complete absence of these cells in CT samples. Next, we examined whether human lung tumor cells produce soluble factors that inhibit differentiation of hematopoietic precursors into mature DC. The addition of small-cell lung carcinoma-conditioned medium to $\mathrm{CD}^{+}{ }^{+}$precursor cell cultures significantly inhibited colony-forming units of DC formation when compared with nontreated control DC cultures. Furthermore, DC generation and differentiation was completely abrogated in $\mathrm{CD34}^{+}$cell cultures treated with CT-conditioned medium, suggesting that CT-derived factors blocked $\mathrm{CD}^{+}{ }^{+}$cell differentiation into DC or induced their apoptosis. Finally, flow cytometry analysis of cultured DC confirmed these results. Thus, analysis of our data suggests that human lung tumors produce factors that inhibit DC generation or maturation and may also induce apoptotic death of DC precursors in vitro.

Copyright (C) 2001 by The United States and Canadian Academy of Pathology, Inc.

VOL. 14, NO. 1, P. 40, 2001 Printed in the U.S.A.

Date of acceptance: July 25, 2000.

Funding: This study was supported by RO1 CA80126 and RO1 CA84270 (to MRS).

Address reprint requests to: Michael R. Shurin, M.D., Ph.D., Immunopathology-CLSI, 5725 CHP-MT, 200 Lothrop Street, Pittsburgh, PA 15213; fax: 412-692-4997.
KEY WORDS: Apoptosis, Bronchial carcinoid tumor, Dendritic cell, Immunohistochemistry, Small-cell lung carcinoma.

Mod Pathol 2001;14(1):40-45

Immune responses to tumor antigens are the result of reciprocal communication between antigenpresenting cells, $\mathrm{T}$ cells, and $\mathrm{B}$ cells. The most potent professional antigen-presenting cells are dendritic cells (DC), which are considered to be solely responsible for initiating primary immune responses, including antitumor immunity $(1,2)$. DC recognize and take up antigen, process it, and present antigenic peptides to $\mathrm{T}$ cells in a major histocompatibility complex (MHC)-restricted manner. Thus, interaction between DC and tumor cells with the tumor tissue, where DC recognize and take up tumor-associated antigens, should play a key role in the induction of specific antitumor immune responses. In fact, it has been shown that the presence of greater numbers of DC within various tumors is often associated with a better prognosis and longer patient survival $(3,4)$.

Varieties of tumor-produced factors exhibit immunomodulatory properties and play an important role in the regulation of tumor growth and escape from immune recognition and elimination. Some of these molecules inhibit natural killer-and cytotoxic $\mathrm{T}$ lymphocyte-mediated cytotoxicity and macrophage functions, induce apoptosis of $\mathrm{T}$ cells and natural killer cells, and cause apoptotic death of DC $(5,6)$. After the identification of DC as Langerhans cells in the epidermis, interstitial cells within nonlymphoid tissues and lymph nodes, and veiled cells in lymph and blood, it was subsequently recognized by clinical pathologists that there was a relationship between the presence and number of DC within the tumor tissue and prognosis.

The identification of DC in the lung and airway tissues of human is based on pleomorphic pathology in conjunction with a high level of MHC Class II expression and lack of expression of characteristic B-cell, T-cell, and macrophage markers. DC express 
variable levels of S-100 protein, CD1a, CD11c, CD83, CD68, RFD1, and a number of adhesion molecules $(4,7)$. The epithelial surfaces of the conducting airways and the deep lung are continuously exposed to various antigens and pathogens from the inhaled air, suggesting that DC in the lung should have specific features caused by the unique lung anatomy and physiology (8). DC are present in the normal airway epithelium, lung parenchyma, and epithelial surface of the lower respiratory tract $(9-11)$. The distribution of DC within the epithelium of conducting airways is similar to the Langerhans cell network in the epidermis (12). The presence of DC in human respiratory tract tissues was also noted in the context of several diseases such as histiocytosis X (13), carcinoma (14), and various granulomatous disorders $(15,16)$. Pulmonary neuroendocrine tumors, in particular carcinoid tumor (CT) and small-cell lung carcinoma (SCLC), are of significant interest because of their ability to secrete a number of hormones and hormone-like substances, peptides, and mediators of inflammation, leading to a specific clinical picture, as well as their ability to cause distant metastases of unpredictable location several months after successful surgery.

A growing body of evidence indicates that tumorinduced immunologic unresponsiveness may be primarily the result of an insufficient number or function of immune cells, including DC $(5,17,18)$. However, to our knowledge, the interaction between DC and tumor cells in the lung has not yet been investigated. The aim of this study was to compare the distribution of DC in human SCLC and CT tissues and determine whether SCLC- and CTderived soluble factors regulate DC generation and maturation. We have demonstrated that CT cells were significantly stronger in elimination of DC from the tumor microenvironment and inhibition of DC generation (dendropoiesis) when compared with SCLC cells. These results might serve as a basis for the further development of immunohistochemical criteria for the differential diagnosis of lung tumors and provide new insights in our understanding of the immunobiology of lung cancer.

\section{MATERIALS AND METHODS}

\section{Immunohistochemistry}

Samples of bronchial CT and SCLC tissues frozen optimal cutting temperature compound were obtained from the University of Pittsburgh Cancer Institute Tumor Tissue Bank. For the staining of human DC, anti-CD1a, anti-CD83, anti-S-100, or anti-human leukocyte antigen-DR antibodies (Immunotech, Westbrook, ME) were used. Staining was performed with horseradish peroxidase technique after acetone- based fixation of the tissue. Rehydration and washing were done with standard phosphate buffered saline (PBS; Gibco BRL, Grand Island, NY) and a 3\% PBS solution of goat serum (Gibco) prepared ex tempore. Antibody binding was localized by biotinylated secondary antibodies followed by an avidin-conjugated horseradish peroxidase and diaminobenzidine $\left(3,3^{\prime}\right.$ diaminobenzidine) substrate with an auidin biotinylated enzyme complex detection kit (Vector Laboratories, Burlingame, CA). Isotype-matched antibodies were used as a negative control. DC infiltration per high power field was graded 0 through 4 as follows: 0 , absence of DC; 1, a small amount of DC (fewer than 10 cells); 2 , a medium infiltration (10 to 50 cells); 3 , a medium to high amount of DC (more than 50 cells); and 4, a high DC number (widely distributed cells throughout the whole slide).

\section{Human DC Cultures}

Human DC were generated from $\mathrm{CD} 34^{+}$precursors isolated from umbilical cord blood and cultured with recombinant human granulocyte-macrophage colonystimulating factor $(1000 \mathrm{U} / \mathrm{mL})$ and recombinant interleukin-4 (1000 U/mL) in AIM-V medium (BioWhitacker, Walkersville, $\mathrm{MD}$ ) supplemented with $2.5 \%$ heatinactivated human type $\mathrm{AB}$ serum (Sigma, St. Louis, MO). Briefly, fresh cord blood samples were diluted 1:4 with PBS, and $25 \mathrm{~mL}$ of cell suspension was loaded onto $15 \mathrm{~mL}$ HistoPrep solution (Sigma). Mononuclear cells were isolated from the interface after centrifugation (400 $\mathrm{g}, 30 \mathrm{~min}, 20^{\circ} \mathrm{C}$ ) and washed twice in PBS. CD34 ${ }^{+}$cells were separated with magnetic cell sorting (MACS, Miltenyi Biotech, Sunnyvale, CA). According to the manufacturer's protocol, mononuclear cells were labeled with QBEND10, and anti-CD34 antibodies attached to the superparamagnetic microparticles. The cell suspension was then passed through a MiniMacs separation column in a highly magnetized field. The percentage of $\mathrm{CD}^{+} 4^{+}$cells was determined in each experiment by a FACScan analysis, and only cell suspensions enriched with up to $90 \%$ and more of CD34 $4^{+}$cells were selected for further experiments. The cell viability was more than $95 \%$ in all experiments.

\section{Tumor Cell Lines}

Bronchial CT and SCLC lines CRL-5815 and CRL5837, respectively, were obtained from American Type Culture Collection (Rockville, MD) and cultured in a complete RPMI 1640 medium supplemented with $2 \mathrm{~mm}$ L-glutamine, $100 \mathrm{U} / \mathrm{mL}$ penicillin, 100 $\mu \mathrm{g} / \mathrm{mL}$ streptomycin, $1 \mathrm{~mm}$ sodium pyruvate, $0.1 \mathrm{~mm}$ nonessential amino acids, and 5\% heat-inactivated fetal bovine serum (all from Gibco). Cell-free supernatants were harvested from nonconfluent cultures, formed into aliquots, and kept frozen at $-20^{\circ} \mathrm{C}$ for further use. Tumor-conditioned medium was added 
to hematopoietic precursor cell cultures $(20 \% \mathrm{v} / \mathrm{v})$ in six-well plates (Falcon, Franklin Lakes, NJ) at Day 1. Control DC cultures and DC cultures pretreated with SCLC, and CT supernatants were collected at different times and analyzed by morphologic assessment and FACScan.

\section{Flow Cytometry}

Evaluation of expression of surface molecules on cultured control and tumor supernatant-treated DC was performed with fluorescein isothiocyanateor phycoerythrin-conjugated antibodies recognizing CD83, CD86, CD80, CD1a, and human leukocyte antigen-DR molecules (PharMingen, San Diego, CA). Flow cytometric analysis of stained cells was performed on a Becton-Dickinson FACScan cytometer by Cell Quest software (Becton-Dickinson, San Diego, CA).

\section{RESULTS}

Immunohistochemical Analysis of Human Tumors

As shown in Table $1, \mathrm{CD}^{+} \mathrm{a}^{+}$and $\mathrm{CD}^{+} 3^{+}$DC were not found in bronchial CT samples $(n=4)$. In SCLC tissues, $\mathrm{CD} \mathrm{a}^{+}$and $\mathrm{CD} 3^{+}$cells were detected with different frequencies $(n=4)$. For instance, one to four $\mathrm{CD} \mathrm{a}^{+}$cells and two to five $\mathrm{CD} 83^{+}$cells were observed per power field in SCLC samples, whereas no positive staining was detected in CT tissues (Fig. 1). Detected DC were distributed mostly in short chains and small clusters within the tumor tissues. Furthermore, S- $100^{+}$cells were detected in very small amounts in both CT and SCLC tissue samples. Next, cells positive for MHC Class II markers were observed in both SCLC and CT tissues in approximately equal quantities. Cells positive for $\operatorname{IgG}_{1}$ were not detected; these served as a control for the specificity of the staining procedure. Interestingly, the distribution of $\mathrm{CDla}^{+}, \mathrm{S}-100^{+}$, or $\mathrm{CD} 83^{+}$DC was different, and no significant correlations between these three DC markers were determined. Analysis of these data may suggest that different populations of DC or DC at different stages of their maturation have different sensitivity to tumor-induced apoptosis or different ability to migrate to the tumor tissues (tumor tropism). Importantly, this effect was tumor specific.
Because DC were practically undetectable in CT specimens, we hypothesized that factors derived from CT cells may block the final steps of DC differentiation within the tumor or cause premature cell death (apoptosis) of DC precursors. To test this hypothesis, we generated DC from $\mathrm{CD} 34^{+}$precursors in vitro in the presence of tumor-derived factors.

\section{Inhibition of DC Maturation by \\ Tumor-Derived Factors}

To evaluate whether CT- or SCLC-derived factors affect DC maturation, DC cultures were coincubated with cell-free tumor cell supernatants. As shown in Figure 2, in SCLC-treated, CD34-derived DC cultures, dendropoiesis was not completely blocked and the formation of DC clusters and colonies was clearly detectable, although the number of generated DC was significantly decreased. In contrast, in CT-treated cultures, differentiation of DC was completely abrogated. Four to 5 days after the initiation of CD34-derived DC cultures coincubated with CT-conditioned medium, only a few live adherent cells were detected, which disappeared during further culture with CT-conditioned supernatant. Thus, these results suggest that generation of DC in CT-treated, CD34-derived cultures was blocked, which was likely caused by the inhibited renewal and differentiation of hematopoietic precursor cells. SCLC-derived factors did not block dendropoiesis but significantly inhibited it in comparison with control nontreated DC cultures.

We found that in the presence of SCLCconditioned medium, cultured DC generated from the $\mathrm{CD}_{3}{ }^{+}$precursor cells displayed significantly different phenotypic characteristics in comparison with DC obtained from control cultures (Table 2). For instance, expression of CD80 molecules decreased from 31.1 to $7.2 \%$ in control cultures and tumor-treated cultures, respectively. Similarly, expression of human leukocyte antigen-DR and B7.2 molecules was inhibited up to 84 and $41 \%$, respectively. Decreased expression of MHC Class II and costimulatory molecules on DC produced in cultures treated with tumor-derived factors suggests that they are likely not mature or activated. No FACScan analysis of CT-treated cultured cells was possible because of extremely low yield of har-

TABLE 1. Immunohistochemical Analysis of Tumor-Associated Dendritic Cells in SCLC and CT Tissues

\begin{tabular}{lcccrrr}
\hline & CDla & CD83 & S-100 & CD4 & HLA-DR \\
\hline SCLC & $2.7 \pm 0.3^{* *}$ & $1.7 \pm 0.6$ & $1.7 \pm 0.6$ & $3.1 \pm 0.1$ & $2.9 \pm 0.2$ \\
CT & 0 & 0 & $0.5 \pm 0.2$ & $1.1 \pm 0.1$ & $3.0 \pm 0.1$ & 0 \\
\hline
\end{tabular}

* IgG was used as an isotype control, as described in Materials and Methods.

** Each number represents mean number \pm SEM of DC per power view determined as described in Material and Methods.

CT, carcinoid tumor; HLA, human leukocyte antigen; Ig, immunoglobulin; SCLC, small-cell lung carcinoma. 


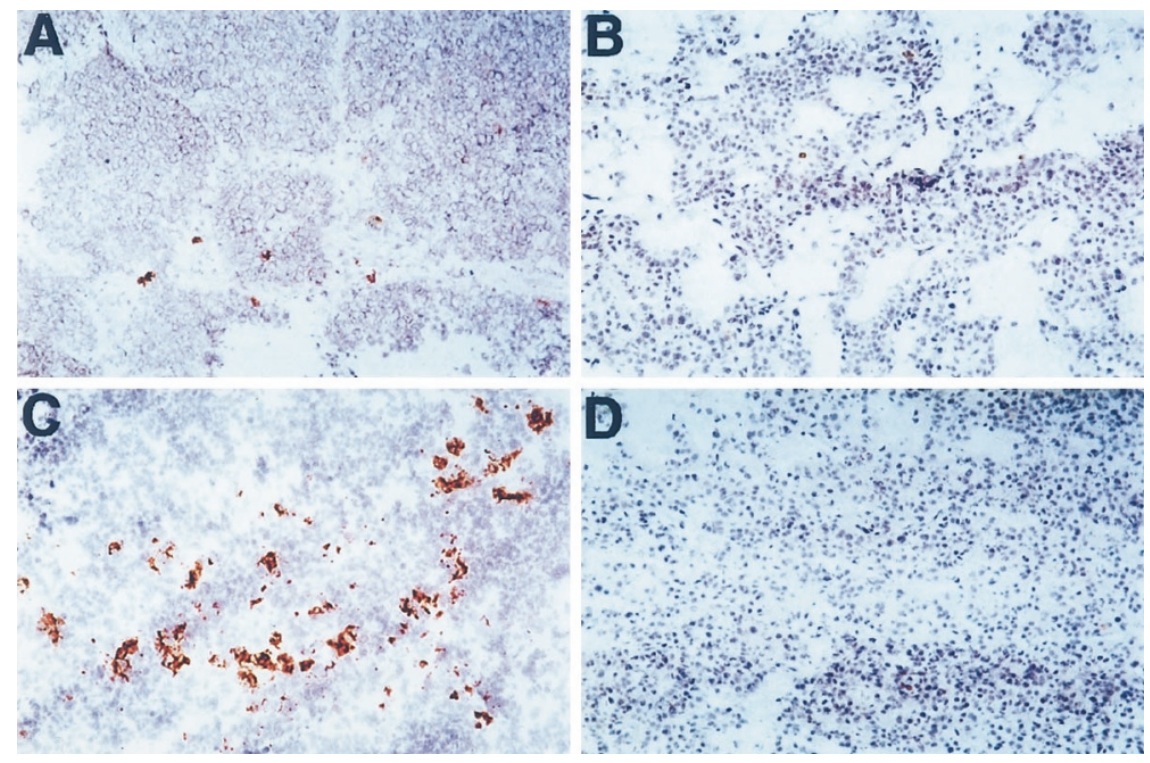

FIGURE 1. Immunohistochemical analysis of tumor-infiltrating dendritic cells (DC) in small-cell lung carcinoma (SCLC) and carcinoid tumor (CT) tissues. Tissue slides $(6 \mu \mathrm{m})$ from optimal cutting temperature frozen tumor samples were stained with DC-related antibodies as described in Materials and Methods. Immunohistochemical analysis of CT (B and D) and SCLC (A and $\mathbf{C})$ tissues revealed the presence of $\mathrm{CD}_{3}{ }^{+}(\mathbf{A})$ and $\mathrm{CDla}^{+}(\mathbf{C})$ DC within SCLC tissues (A and B) but not CT tissues $(\mathbf{C}$ and D). Cells positive for S-100 and major histocompatibility complex Class II markers were found in both SCLC and CT tissues in approximately equal quantities.
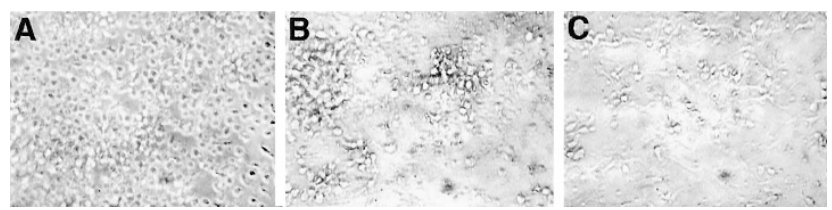

FIGURE 2. Small-cell lung carcinoma (SCLC) and carcinoid tumor (CT) inhibited colony formation and generation of dendritic cells (DC) in CD34-derived cultures in vitro. $\mathrm{CD} 34^{+}$cells were isolated from umbilical cord blood and cultured in granulocyte-macrophage colonystimulating factor and interleukin-4-supplemented medium as described in Materials and Methods. Colony formation was evaluated by light microscopy in control cultures and in cultures treated with SCLC or CT conditioned media. SCLC-derived factors significantly inhibited formation of colony-forming units of DC, whereas CT-derived factors resulted in a complete abrogation of colony formation. The experiment was repeated twice with similar results. A, control nontreated DC cultures at Day 7. B, SCLC-treated DC cultures. C, CTtreated DC cultures.

vested cells. Taken together, these results demonstrate that the generation of DC from $\mathrm{CD} 34^{+}$precursors can be blocked or markedly suppressed by the presence of lung tumor cells.

In summary, we have demonstrated here that human CT tumors were significantly less infiltrated by DC than SCLC tumors and that CT-derived factors inhibited the differentiation of $\mathrm{CD}_{3} 4^{+}$hematopoietic precursors into DC significantly more strongly than SCLC-derived factors in vitro.

\section{DISCUSSION}

CT and SCLC belong to the so-called pulmonary neuroendocrine tumors. Bronchial carcinoid tu- mors consist of the typical carcinoid and atypical carcinoid tumors with the latter having more malignant histologic and clinical characteristics (19). For instance, carcinoid tumors are distinguished from morphologically similar atypical carcinoid tumors by the presence of necrotic and mitotic cells (20). CT secrete substances with hormonal activity, including anti-diuretic hormone, cortisol, bombesine-like factors, and adrenocorticotropin, are insensitive to radiation therapy and poorly responsive to traditional chemotherapy, and they generate distant metastases after successful surgery, along with signs of carcinoid disease. Typically, CT metastasizes to lymph nodes, liver, and bones.

In contrast to CT, SCLC is characterized by a rapid growth pattern with an aggressive clinical course and early appearance of widespread distant metastases. It accounts for 20 to $25 \%$ of lung cancer, and approximately 28,000 new cases occur in the United States each year. High sensitivity to radiation therapy and prompt response to antitumor agents is typical for the SCLC. Bronchial CT is often confused with SCLC, and the correct diagnosis requires electron microscopy and immunohistochemical studies, in addition to light microscopic analysis $(20,21)$. Distinction between CT and SCLC is often difficult from a single microscopic analysis of hematoxylin and eosin-stained sections. The importance of distinguishing between CT and SCLC is underlined by the fact that CT and SCLC require a different approach to therapy. 
TABLE 2. Phenotypic Characteristics of Control and Tumor-Treated Dendritic Cell Cultures

\begin{tabular}{|c|c|c|c|c|c|c|}
\hline & CD1a & CD80 & CD83 & $\mathrm{CD} 40$ & CD86 & HLA-DR \\
\hline Control & $87.4 \% *$ & $31.1 \%$ & $13.2 \%$ & $51.6 \%$ & $16.5 \%$ & $77.8 \%$ \\
\hline SCLC & $18.0 \%$ & $7.2 \%$ & $11.3 \%$ & $7.3 \%$ & $9.7 \%$ & $12.3 \%$ \\
\hline $\mathrm{CT}$ & ND & ND & ND & ND & ND & ND \\
\hline
\end{tabular}

* The percentage of positive cells determined by FACScan analysis as described in Materials and Methods.

ND, nondetectable; HLA, human leukocyte antigen.

Tumor growth and progression is regulated in part by the development of specific antitumor immune responses. Immunologic recognition of tumor antigens has been subsequently documented in patients with different malignancies. However, these responses are often muted and ineffective in eradicating disease. The development of immune tolerance toward malignant cells is partly because tumor antigens are presented to the immune system in the absence of costimulatory signals crucial for the initiation of effective T-cell responses. Therefore, $\mathrm{T}$ cells with the capability to recognize these antigens become anergic (22). It is possible that CT induce high levels of immune tolerance attributable to the absence of antigen-presenting cells. In fact, we have demonstrated here that CT tissues contained only a single DC per power view, as determined by the immunohistochemical analysis. The absence of tumor-infiltrating DC within the CT tissues may also explain the absence of immune memory, resulting in the formation of distant metastases after the surgical intervention in patients with CT.

The key mechanism of tumor-associated immunosuppression is the inhibition of immune effector cells at the tumor site, including $\mathrm{T}$ cells and macrophages $(18,23)$. There is increasing evidence that function and activity of DC, responsible for the induction of antitumor immunity, can also be modified by tumor-derived factors $(5,24)$. Consequently, impairment of DC number or activity might result in a deficient activation and expansion of specific T lymphocytes. In fact, it has been recently reported that human $\mathrm{CD}^{+} 3^{+}$DC obtained from progressing chemotherapy-resistant melanomas reveal a marked down-regulation of CD86 molecules and induced anergy in syngeneic $\mathrm{CD}^{+} \mathrm{T}$ cells (25). The inability of tumor-associated DC to effectively activate $\mathrm{T}$ cells may explain why tumorinfiltrating lymphocytes fail to eliminate tumor cells. Furthermore, coincubation of both murine and human DC with tumor cells resulted in a significant inhibition of their ability to stimulate T-cell proliferation (6). However, the mechanism and tumor-derived factors responsible for the inhibition of the immune system are not yet elucidated.

It has been shown that a number of tumors, including lung cancer, express functional Fas ligand (CD95L), which is a counterpart for the Fas receptor
(CD95) expressed on virtually all mammalian cells, including activated $\mathrm{T}$ cells within tumor tissues (26-28). This suggests that tumor cells might induce cell death of neighboring T cells, and Fas/Fas ligand-mediated apoptosis of tumor-infiltrating lymphocytes might contribute to the maintaining of immune privilege at the tumor site (29). Because DC express high levels of Fas, it is possible that a similar mechanism may be involved in the elimination of tumor-associated DC as well $(5,30)$. Interestingly, we have demonstrated here striking differences in the number of tumor-infiltrating DC between CT and SCLC tissues. It is likely that different tumor-derived factors have different effects on DC survival within the tumor. Although the exact mechanism of tumor-induced apoptosis of DC is unclear, it has been shown that direct contact between DC and tumor cells results in a faster appearance of apoptotic figures when compared with the induction of DC apoptosis by soluble tumorderived factors. It is likely that both soluble and membrane-bound molecules are involved in this phenomenon. Different tumor cells may release and express on the surface different proapoptotic factors, such as NO, transforming growth factor $\beta$, interleukin-10, gangliosides, Fas ligand, TNFrelated apoptosis-inducing ligand and others. The roles of these factors, as well as the role of the intracellular signal transduction pathways responsible for tumor-induced apoptosis in DC, remain to be determined (5).

Regulation of the functional phenotype of lung and airway DC, as well as DC survival, depends on the local cytokine and hormone network. For instance, DC can be modulated by endogenous or exogenous steroids, and it has been hypothesized that up-regulation of the antigen presenting function of airway DC in chronically treated patients may be an essential part of the pathogenesis of atopic asthma (31). Furthermore, steroid-treated DC lose their capacity to take up and process newly encountered antigens (32). It is possible that secretion of cortisol-like substances by CT cells might explain the phenomenon of tumor-induced inhibition of DC generation and survival demonstrated in our study. It is also possible that the suppression of DC is responsible for the CT cells' escape from immune recognition in the blood stream and non- 
lymphoid tissues after successful surgery and, subsequently, metastatic disease.

Finally, we demonstrated that DC maturation and function are inhibited by both SCLC and CT cells. However, the mechanism of this phenomenon is likely to be different for both types of tumors. SCLC derives a number of soluble factors, leading to functional suppression of DC, which has been shown by immunohistochemical and flow cytometry analysis. CT produces soluble factors, causing the high apoptotic rate of DC precursors in cultures. In addition, the evaluation of DC number and distribution might be used for prognosis and monitoring biopsies in order to evaluate the effect of current therapy and to predict the progression of primary or metastatic tumors.

\section{REFERENCES}

1. Shurin MR. Dendritic cells presenting tumor antigen. Cancer Immunol Immunother 1996;43:158-64.

2. Banchereau J, Steinman RM. Dendritic cells and the control of immunity. Nature 1998;392:245-52.

3. Becker Y. Anticancer role of dendritic cells (DC) in human and experimental cancers-a review. Anticancer Res 1992; 12:511-20.

4. Austin JM. The dendritic cell system and anti-tumor immunity. In Vivo 1993;7:193-202.

5. Shurin MR, Esche C, Lokshin A, Lotze MT. Apoptosis in dendritic cells. In: Lotze MT, Thomson AW, editors. Dendritic cells: biology and clinical applications. San Diego, CA: Academic Press: 1999;1999:673-92

6. Esche C, Lokshin A, Shurin GV, Gastman BR, Rabinowich H, Watkins SC, et al. Tumor's other immune targets: dendritic cells. J Leukoc Biol 1999;66:336-44.

7. Hart DNJ. Dendritic cells: unique leukocyte populations which control the primary immune response. Blood 1997;90: 3245-87.

8. McWilliam AS, Nelson DJ, Holt PG. The biology of airway dendritic cells. Immunol Cell Biol 1995;73:405-13.

9. Richard S, Barbey S, Pfister A, Scheinmann P, Jaubert F, Nezelof C. Demonstration of Langerhans cells in the human bronchial epithelium. C R Acad Sci III 1987;305:35-9.

10. Soler P, Moreau A, Basset F, Hance AJ. Cigarette smokinginduced changes in the number and differentiated state of pulmonary dendritic cells/Langerhans cells. Am Rev Respir Dis 1989;139:1112-7.

11. van Haarst JM, Hoogsteden HC, de Wit HJ, Verhoeven GT, Havenith CE, Drexhage HA. Dendritic cells and their precursors isolated from human bronchoalveolar lavage: immunocytologic and functional properties. Am J Respir Cell Mol Biol 1994;11:344-50.

12. Holt PG, Schon-Hegrad MA, Phillips MJ, McMenamin PG. Ia-positive dendritic cells form a tightly meshed network within the human airway epithelium. Clin Exp Allergy 1989; 19:597-601.
13. Soler P, Chollet S, Jacque C, Fukuda Y, Ferrans VJ, Basset F. Immunocytochemical characterization of pulmonary histiocytosis X cells in lung biopsies. Am J Pathol 1985;118:439-51.

14. Hammar S, Bockus D, Remington F, Bartha M. The widespread distribution of Langerhans cells in pathologic tissues: an ultrastructural and immunohistochemical study. Hum Pathol 1986;17:894-905.

15. Webber D, Tron V, Askin F, Churg A. S-100 staining in the diagnosis of eosinophilic granuloma of lung. Am J Clin Pathol 1985;84:447-53.

16. Munro CS, Campbell DA, Du Bois RM, Mitchell DN, Cole PJ, Poulter LW. Dendritic cells in cutaneous, lymph node and pulmonary lesions of sarcoidosis. Scand J Immunol 1987;25: 461-7.

17. Whiteside TL, Jost LM, Herberman RB. Tumor-infiltrating lymphocytes. Potential and limitation to their use for cancer therapy. Crit Rev Oncol Hematol 1992;12:24-47.

18. Wojtowicz-Praga S. Reversal of tumor-induced immunosuppression: a new approach to cancer therapy [see comments]. J Immunother 1997;20:165-77.

19. Arrigoni MG, Woolner LB, Bernatz PE. Atypical carcinoid tumors of the lung. J Thorac Cardiovasc Surg 1972;64:413-21.

20. Franklin WA. Pathology of lung cancer. J Thorac Imaging 2000;15:3-12.

21. Zhang Z, Li S, Ge F, Li Z, Sun C, Xu L, et al. Surgical treatment and prognosis of bronchial carcinoid tumor. Chin Med Sci J 1996;11:248-51.

22. Avigan D. Dendritic cells: development, function and potential use for cancer immunotherapy. Blood Rev 1999;13:5164.

23. Kavanaugh DY, Carbone DP. Immunologic dysfunction in cancer. Hematol Oncol Clin North Am 1996;10:927-51.

24. Shurin MR. Regulation of dendropoiesis in cancer. Clin Immunol Newslett 1999;19:135-9.

25. Enk AH, Jonuleit H, Saloga J, Knop J. Dendritic cells as mediators of tumor-induced tolerance in metastatic melanoma. Int J Cancer 1997;73:309-16.

26. Hahne M, Rimoldi D, Schroter M, Romero P, Schreier M, French LE, et al. Melanoma cell expression of Fas(Apo-1/ CD95) ligand: implications for tumor immune escape. Science 1996;274:1363-6.

27. Niehans GA, Cherwitz DL, Staley NA, Knapp DJ, Dalmasso AP. Human carcinomas variably express the complement inhibitory proteins CD46 (membrane cofactor protein), CD55 (decay-accelerating factor), and CD59 (protectin). Am J Pathol 1996;149:129-42.

28. O'Connell J, O'Sullivan GC, Collins JK, Shanahan F. The Fas counterattack. Fas-mediated T cell killing by colon cancer cells expressing Fas ligand. J Exp Med 1996;184:1075-82.

29. Griffith TS, Ferguson TA. The role of FasL-induced apoptosis in immune privilege. Immunol Today 1997;18:240-4.

30. Lu L, Qian S, Hershberger PA, Rudert WA, Lynch DH, Thomson AW. Fas ligand (CD95L) and B7 expression on dendritic cells provide counter- regulatory signals for T cell survival and proliferation. J Immunol 1997;158:5676-84.

31. Holt PG. Macrophage: dendritic cell interaction in regulation of the IgE response in asthma. Clin Exp Allergy 1993;23:4-6.

32. Holt PG, Thomas JA. Steroids inhibit uptake and/or processing but not presentation of antigen by airway dendritic cells. Immunology 1997;91:145-50. 